\title{
Origine et progression des tumeurs épithéliales : vers les mécanismes cellulaires et moléculaires
}

David

Wynford-Thomas

\section{ADRESSE ET TIRÉS À PART}

D. Wynford-Thomas : professeur en pathologie moléculaire. CRC Thyroid Tumour Biology Research Group, Department of Pathology, University of Wales College of Medicine, Heath Park, Cardiff CF4 $4 \times N$,

Deux catégories d'événements moléculaires semblent être des initiateurs possibles de la tumorigenèse thyroïdienne : des mutations de molécules transductrices du signal de prolifération et de différenciation, sous-unité $\alpha_{\mathrm{s}}$ de la protéine $G_{\mathrm{s}}$ et $\mathrm{p} 21^{\text {ras }}$; et des réarrangements de gènes codant pour des récepteurs tyrosine kinases $R E T$ et $T R K$. Les premières anomalies conduisent d'abord à des tumeurs bénignes progressant ensuite vers des cancers vésiculaires, alors que les secondes semblent aboutir directement à des cancers papillaires. Ce schéma de tumorigenèse d'un tissu à renouvellement conditionnel pourrait s'opposer à celui de tissus habituellement prolifératifs, comme le côlon. Dans ces cas, en effet, les cellules prolifératives engendrent des cellules filles dont la moitié est programmée à se différencier et à mourir. Le développement d'une tumeur exige, par conséquent, des événements bloquant la différenciation et/ou l'apoptose, et non pas simplement une augmentation du potentiel prolifératif.

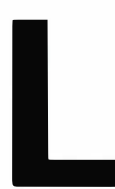

es tissus épithéliaux représentent le site majeur de la carcinogenèse humaine, et sont à l'origine de plus de $90 \%$ des cancers [1], dont ceux du poumon, du sein, du côlon et de l'estomac.

Malgré cette importance clinique, les cellules épithéliales ont été relativement peu étudiées sur un plan fondamental, par rapport aux cellules mésenchymateuses. Cela s'explique par les difficultés pratiques qui entravent (encore aujourd'hui) l'utilisation de nombreux tissus épithéliaux in vitro - faible survie en culture primaire, résistance à la transformation, inefficacité du transfert de gène...
Ces dernières années, une approche différente est devenue possible en court-circuitant ces difficultés par la mise en évidence directe des anomalies génétiques dans une tumeur à l'aide des techniques de biologic moléculaire - notamment l'analyse par hybridation (Southern blot, Northern blot...) et par séquençage. En particulier, l'augmentation de la sensibilité apportée par la technique de PCR [2] a permis d'effectuer de multiples analyses sur dc petits échantillons, même après fixation au formol. Ainsi, de nombreuses anomalies génétiques ont été décelées dans plusieurs types de tumeurs épithéliales [3]. 
Les bases génétiques de la tumorigenèse épithéliale sont donc abordables, mais une question potentiellement plus difficile reste à résoudre : comment ces anomalies géniques expliquent-elles le comportement anormal observé au niveau anatomopathologique? Autrement dit, quels sont les liens biochimiques entre génotype et phénotype tumoral ?

La plupart des recherches fondamentales sur cette question utilisent (encore pour des raisons pratiques) des modèles tels que les lignées de fibroblastes de souris. Dans l'interprétation de ces analyses, on a tendance à négliger la complexité de l'organisation cellulaire de la majorité des tissus épithéliaux, en considérant la cellule cible presque de la même façon qu'une cellule de levure !

Cette revue tente de faire une analyse moléculaire de la tumorigenèse épithéliale en tenant compte de l'importance de ce contexte tissulaire.

\section{Le modèle thyroïdien}

L'utilisation de populations homogènes, dont chaque mitose engendre deux cellules filles identiques, sans aucune mort cellulaire ni différenciation "terminale ", facilite l'étude de la prolifération cellulaire. Dans ce cas, la croissance cst mathématiquement exponentielle, à une vitesse qui ne dépend que de la durée du cycle cellulaire. Le problème du contrôle du nombre des cellules est donc réduit à la régulation du cycle cellulaire par les facteurs extracellulaires stimulateurs et inhibiteurs.

C'est le cas, in vitro, des lignées de fibroblastes de souris (telles que les cellules $3 \mathrm{~T} 3$ ) et de certaines cellules humaines normales à un stade précoce, dont le temps de doublement peut varier de moins d'un jour à l'infini en fonction de la concentration des facteurs de croissance dans le milieu. In vivo, les cellules qui se rapprochent le plus de ce modèle simpliste sont les épithéliums des parenchymes glandulaires, notamment du foie et des glandes endocrines, dont l'état habituel de prolifération est presque nul mais reste fortement stimulable. Parmi ces tissus cinétiquement "stables" (ou encore appelés de "renouvellement conditionnel ") [4, 5], l'exemple le plus

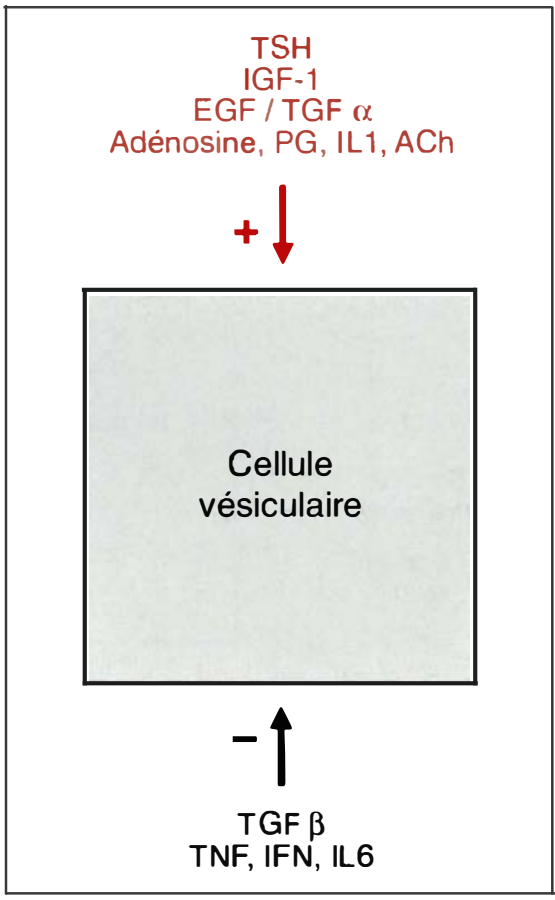

Figure 1. Régulation de la prolifération des cellules épithéliales vésiculaires thyroïdiennes par des facteurs extracellulaires. L'état prolifératif est la résultante de stimuli positifs et négatifs. PG : prostaglandine; IL : interleukine ; ACh : acétylcholine ; TNF : tumor necrosis factor; IFN : interféron.

simple est sans doute la glande thyroïde [6].

\section{Prolifération normale}

Mécanismes de contrôle extracellulaire

Chez l'adulte, les cellules vésiculaires thyroïdiennes ont un temps de renouvellement estimé à plus de 8 ans [7], avec une activité mitotique presque indétectable [8]. Pourtant, elles conservent leur sensibilité aux stimuli physiologiques et pathologiques. Ainsi, chez le rat, une augmentation de la concentration extracellulaire de la thyrotropine (TSH) - provoquée par l'administration d'une substance goitrogène - augmente l'index mitotique jusqu'à 50 fois le taux normal [8]. Un effet similaire chez l'homme rend compte du développement d'un goitre, par exemple dans la maladie de Basedow en réponse aux anticorps qui miment l'action de la TSH [9]. Le contrôle physiologique s'exerce aussi par l'intermédiaire de facteurs inhibiteurs. Bicn que fort difficile à mettre en évidence in vivo, lcs cxpériences in vitro [10] montrent que la prolifération des cellules vésiculaires (comme probablement n'importe quelle cellule épithéliale) est très sensible à l'inhibition par la protéine omniprésente (et mal nommée) TGF $\beta$ (transforming growth factor $\beta$ ) [11].

Au total, à l'état normal il existe un équilibre entre stimulateurs et inhibiteurs (figure 1) qui s'établit juste audessus de zéro. Une augmentation de ces stimulateurs d'origine exogène (ou théoriquement une baisse des inhibiteurs, sans qu'on connaisse d'exemples jusqu'ici), provoquera une prolifération, peut-être inopportune mais réversible - autrement dit, une hyperplasie. En revanche, un déséquilibre d'origine endogène (génétique) est irréversible et donc capable de déclencher un néoplasme. Cela explique l'importance capitalc des voies de signalisation intracellulaires.

\section{Mécanismes de contrôle intracellu-} laire

Bien qu'il nous manque toujours certains détails, la transmission des signaux mitogènes de l'extérieur de la cellule jusqu'au noyau se fait par les étapes suivantes (figure 2) :

- en se fixant sur son récepteur, le facteur de croissance induit un changement de la conformation de la partie interne de ce dernier;

- cela active une voie de signalisation qui aboutit à l'activation d'une ou plusieurs protéine kinases ;

- ces enzymes activent à leur tour d'autres kinases en une véritable cascade ;

- l'effet ultime est la modification, par phosphorylation, de l'activité d'une série de facteurs transcriptionnels qui mettent en jeu le programme génique qui effectuera la réplication de l'ADN et les autres éléments de la division cellulaire.

Les liens entre récepteur et kinase peuvent être plus ou moins directs, à savoir :

- activité kinase intrinsèque du récepteur stimulé dans le cas des récepteurs à tyrosine kinase [12] comme celui de l'EGF (epidermal growth factor), par exemple ;

- activation par fixation directe d'une kinase en ce qui concerne le récep- 


\section{RÉFÉRENCES}

1. Wright NA, Alison M. Biology of Epithelial Cell Populations, vol. 1. Oxford : Clarendon Press, 1984 : 3-4.

2. Wright P, Wynford-Thomas D. The polymerase chain reaction : miracle or mirage ? A critical review of its uscs in diagnosis and rescarch. I Pathol $1990 ; 162$ : 99-117.

3. Wynford-Thomas D. Oncogenes and antioncogenes : the molecular basis of tumour bchaviour. J Pathol 1991; 165 : 187-201.

4. Leblond CP. Classification of ccll populations on the basis of their proliferative activity. J Pathol $1964 ; 14$ : 119-49.

5. Wright NA, Alison M. Biology of Epithelial Cell Populations, vol. 1. Oxford : Clarendon Press, 1984 : 30-1.

6. Smith P, Wynford-Thomas D. Control of thyroid follicular cell proliferation : cellular aspects. In : Wynford-Thomas D, Williams ED, eds. Thyroid Tumours Molecular Basis of Pathogenesis. Edinburgh : Churchill Livingstone, 1989 : 66-90.

7. Coclet J, Fourcau F, Ketclbant P, Galand P, Dumont JE. Cell population kinetics in dog and human adult thyroid. Clin Endocrinol 1989 ; 31 : 655-65.

8. Wynford-Thomas D, Stringer BMJ, Williams ED. Dissociation of growth and function in the rat thyroid during prolonged goitrogen administration. Acta Endocrinologica 1982 ; 101 : 210-6.

9. Mariotti S, Pinchera A. Role of the immune system in the control of thyroid function. In : Greer MA, ed. The Thyroid Gland. New York : Raven Press, 1990 : 147-219.

10. Wyllic FS, Dawson T, Bond JA, et al. Correlated abnormalitics of transforming growth factor-bcta-1 response and p53 expression in thyroid epithclial ccll transforming. Mol Cell Endocrinol 1991 ; 76 : 13-21.

11. Sporn MB, Roberts AB. TGF-beta : problems and prospects. Cell Reg 1990; 1 : 875-82.

12. Ullrich A, Schlessinger J. Signal transduction by receptors with tyrosine kinase activity. Cell $1990 ; 61$ : 203-12.

13. Cantley LC, Auger K R, Carpenter C, et al. Oncogencs and signal transduction. Cell 1991; 64: 281-302.

14. Dumont JE, Jauniaux JC, Roger PP. The cyclic AMP-mediated stimulation of cell proliferation. Trends Biochem Sci 1989 ; 14 :

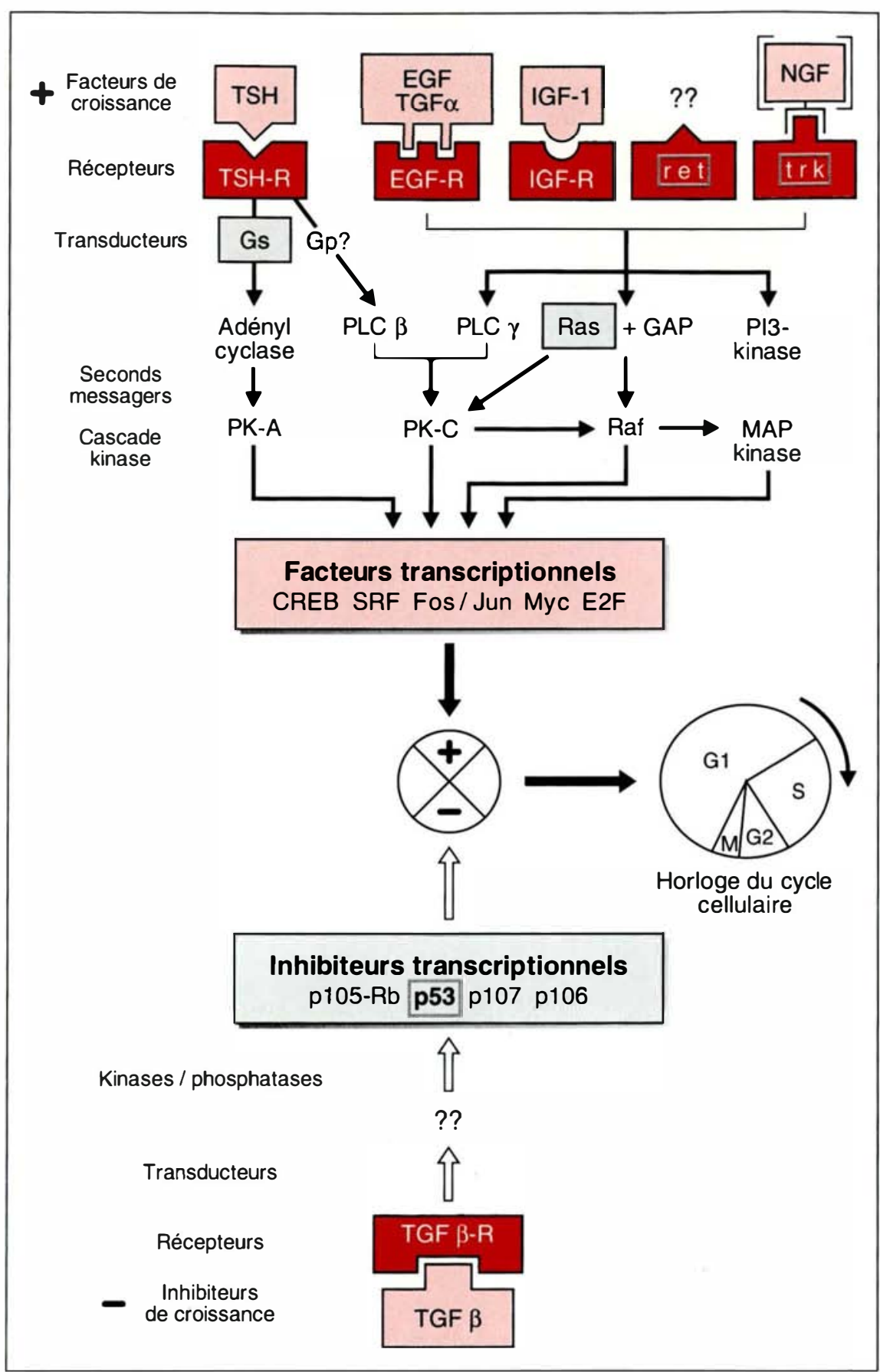

Figure 2. Régulation au niveau intracellulaire. Diverses voies de signalisation transmettent vers le noyau les signaux positifs et négatifs induits par les facteurs extracellulaires. L'interaction entre ces signaux détermine l'activité transcriptionnelle d'une série de gènes clés qui contrôlent le déclenchement des processus de réplication de l'ADN et la division cellulaire (représenté ici comme un oscillateur intrinsèque, horloge du cycle cellulaire). Les éléments connus jusqu'ici comme étant des cibles d'altérations structurales géniques dans les tumeurs thyroïdiennes sont indiqués par des cases tramées grises. A noter que deux d'entre eux - RET et TRK - codent pour des récepteurs qui ne jouent probablement aucun rôle dans la cellule vésiculaire normale. 
teur du lymphocytc $T$ (TCR) fixant c-Fyn, un membre de la famille Src [13] ;

- activation indirecte d'une kinase via des signaux intcrmédiaires (encore appelés "seconds messagers"), comme c'est lc cas pour le récepteur de la TSH, la protéine kinase A ct le second messager AMP cyclique (AMPc) [14].

Les récepteurs peuvent être classés en fonction de ces propriétés, les cellules vésiculaires thyroïdiennes en possédant deux classes : les récepteurs à activité tyrosine kinasc (T/C) ct ceux utilisant obligatoirement des seconds messagers.

Récepteurs à activité tyrosine kinase Parmi diverses cibles, l'activité tyrosine kinase provoque l'autophosphorylation du récepteur lui-même, qui (selon les cas) lui permet de fixer ct donc d'activer d'autres kinases (comme celles de la famille $\mathrm{Src}$ ) et aussi des enzymes telles que la phospholipasc C- $\gamma$ et la phosphatidylinositol(PI)-3-kinasc [13, 15]. Ces dernières engendrent des messagers sccondaires de la voie PI [15] qui aboutissent à l'activation d'une importante kinasc à sérine-thréonine, la protéine kinase C (PK-C). Par ailleurs, ces récepteurs sont capables, par divers mécanismes moins connus, d'activer une voie clé qui passc par la protéine $\mathrm{p} 21^{\text {ras }}\left(\mathrm{m} / \mathrm{s} \mathrm{n}^{\circ} 5\right.$, vol. 8 , p. 471) [16] (voir ci-dessous) et qui, elle aussi, mène à l'activation de nombreuses sérine-thréoninc kinases (figure 2).

Actuellement, il existe deux récepteurs appartenant à cette classe dont le rôle des ligands (physiologique ou pathologique) a été bien établi pour la thyroïde : ceux de l'EGF [17] (ou de son analogue TGF $\alpha$ ) et de l'IGF-1 (insulin-like growth factor 1) [18]. Chacun ne possède qu'une partie des propriétés décrites ci-dessus, assurant ainsi unc spécificité partielle de signalisation et expliquant l'interaction synergique entre l'EGF et l'IGF-1 (voir ci-dessous). Il est probable que l'HGF (hepatocyte growth factor) et des membres de la famille FGF (fibroblast growth factors) sont aussi impliqués [19].

\footnotetext{
Récepteurs utilisant obligatoirement des seconds messagers

Le récepteur de la TSH [20] est

l'archétype de cette catégorie. Dénué d'activité enzymatique lui-même, le réccpteur stimule l'enzyme adénylatc cyclase par l'intermédiaire d'un transducteur - une protéine $G\left(G_{s}\right)$. Le second messager qui en résulte $\mathrm{AMPc}$ - active à son tour la protéine kinase de cette voie - la PK-A. A une plus forte concentration de $\mathrm{TSH}$, une deuxic̀me voie est mise en jeu [21] qui passe par une autre protéine $G$ pour activer une enzyme, la PL-C $\beta$, qui est apparentée à la formc $\gamma$, activée directement par les récepteurs à $\mathrm{TK}$, et qui engendre les mêmes messagers : le diacylglycérol (DAG) qui stimule la PK-C, et l'inositol trisphosphate (IP3) qui déclenche une augmentation de la concentration intracellulaire en $\mathrm{Ca}^{2+}$. Cette voie est aussi empruntée par toute une gamme de petits ligands (adénosine, ATP, TRH...) dont la signification cst loin d'êtrc claire.

\section{- Pourquoi une telle diversité de voies mitogènes?}

D'abord, la cellule normale nc répond pas à un seul facteur de croissance, mais à des combinaisons obligatoires - dans la cellule vésiculairc, par exemple, l'IGF-1 doit être présent pour permettre une réponse à la TSH [18] ou à l'EGF. Évidemment, l'existence de voies spécifiqucs permet de telles interactions synergiques, bien que l'on ne connaisse pas encore les bascs biochimiques précises (multiples modifications d'une molécule clé comme c-Jun ou c-Fos?). Ensuite, cela permct unc divcrsité des réponses associées à la prolifération. Par exemplc, bien que la TSH et l'EGF stimulent tous les deux la prolifération de la cellule vésiculaire, ils ont des effets opposés sur la différenciation (synthèse de la thyroglobuline par exemple), la TSH (via l'AMPc) étant stimulatrice, l'EGF inhibiteur. Enfin, cela permet la modulation de la sensibilité à des facteurs individuels. Ainsi, dans la thyroïde de rat lors d'un traitement prolongé par un goitrogène, il existe une désensibilisation à la TSH, mais aucune diminution de sa réponse proliférative aux stimuli "réparateurs " engendrés par unc incision chirurgicale [22].

Un réseau analogue existe pour relayer les signaux antimitogènes des récepteurs de facteurs inhibiteurs, telle la famille TGF $\beta$. De plus, on retrouve le mêmc principe de phosphorylation de protéines clés, notamment la protéinc p105 ${ }^{\mathrm{Rb}}$ [23] (produit de l'anti-oncogène $R b$, gène de susccptibilité du rétinoblastome) qui influe sur le programme transcriptionnel, mais dans le sens opposé. D'après des donnécs récentes, cettc inhibition se fait soit par blocage de la synthèse des facteurs transcriptionnels "positifs" (commc les protéines c-Myc et E2F), soit par lcur fixation dirccte, soit par blocage de leur sitc cible [24]. Malheureuscment, des données spécifiques concernant les cellules vésiculaires dans ce domaine manquent actucllement.

\section{Prolifération néoplasique}

En principe, un néoplasme est la conséquence de n'importe quelle anomalie qui perturbe de façon permanente l'équilibre entrc les signaux intracellulaires décrits ci-dessus - soit par augmentation des signaux positifs, soit par atténuation des voies inhibitrices. En pratique, cependant, il semble que le nombre de tclles anomalies soit assez restreint dans les tumeurs thyroïdiennes, comme dans d'autres types de tumeurs. Cela suggère que la plupart des anomalies potentielles pourraient êtrc compensées par le réseau de signalisation, ct quc celles qui sont sélectionnées dans les tumeurs représentent donc les "points faibles" du systèmc. Jusqu'à présent, dans la thyroïde, quatre anomalies géniques aboutissant à l'activation de signaux positifs, et une seule inactivation de signaux négatifs, ont été miscs en évidence (figure 2).

\section{Activation des voies stimulatrices :} oncogènes activés

L'augmentation anormale de l'activité d'une protéine stimulatrice de la croissance (produit d'un oncogène) se fait soit par une altération structurale (mécanisme qualitatif) soit par surexpression (mécanisme quantitatif) [3]. La thyroïde présente des exemples de ces deux mécanismes : activation de deux gènes codant pour des protéines transductrices (RAS [25] et GSP [26]) par mutations ponctuelles des séquences codantes, ct activation de deux gènes codant pour des récepteurs (RET [27] et TRK [28]) par une combinaison d'altération structurale et de sur-expression. (Cela doit 


\section{RÉFÉRENCES}

15. Berridge MJ, Irvine RF. Inositol phosphates and cell signalling. Nature 1989 ; 341: 197-205.

16. McCormick F. The world according to GAP. Oncogene $1990 ; 5: 1281-3$.

17. Roger $P$, Taton $M$, Van Sande J, Dumont JE. Mitogenic effects of thyrotropin and adenosine 3,5'-monophosphate in differentiated normal human thyroid cells in vitro. J Clin Endocrinol 1988 ; 66 : 1158-65.

18. Williams DW, Williams ED, WynfordThomas D. Loss of dependence in IGF-1 for proliferation of human thyroid adenoma cclls. $\mathrm{Br} J$ Cancer 1988 ; 57 : 535-9.

19. Bond JA, Graham GJ, Freshney M, et al. Detection and partial purification of a potent mitogenic factor for human thyroid follicular cells. Mol Cell Endocrinol 1992 ; 84 : R15-21.

20. Vassart G, Parmenticr M, I ibert F, Dumont J. Le réceptcur de la thyrotropine : un membre pas comme les autres de la famille des réceptcurs couples aux protéines G. médecine/sciences $1990 ; 6$ : 985-90.

21. Laurent E, Mockel J, Van Sande J, Graff I, Dumont JE. Dual activation by thyrotropin of the phospholipase $\mathrm{C}$ and cyclin AMP cascades in human thyroid. Mol Cell Endocrinol 1987 ; 52 : 163-6.

22. Wynford-Thomas D, Stringer BMJ, Harach HR, Williams ED. Control of growth in the rat thyroid: an cxample of specific desensitisation to trophic hormone stimulation. Experientia 1983 ; 39 : 421-3.

23. Pictonpohl JA, Stcin RW, Moran A, et al. TGF-bcta-1 inhibition of c-myc transcription and growth in keratinocytes is abrogated by viral transforming proteins with $\mathrm{pRB}$ binding domains. Cell 1990; 61 : 777-85.

24. Wagner S, Green MR. Retinoblastoma : a transcriptional trys. Nature 1991 ; 352 : 189-90.

25. Bos JI. Ras oncogenes in human cancer : a revicw. Cancer Res 1989 ; 49 : 4682-9.

26. Bourne HR, Sanders DA, McCormick F. The GTPasc supcrfamily : conserved structure and molecular mechanism. Nature 1991 ; 349 : 117-27.

27. Gricco M, Santoro M, Berlingieri MT, et al. PTC is a novel rearranged form of the ret proto-oncogenc and is frequently detected in vivo in human thyroid papillary car- être distingué de la surexpression de nombreux autres gènes sans aucun défaut primaire dans le gène luimême - par exemple ERBB [29], $E R B B 2$ [30] et $C-M Y C$ [31] dans les cancers thyroïdiens - , qui correspondent vraisemblablement à des changements secondaires à la prolifération néoplasique.)

\section{Activation de transducteurs}

- Mutation du gène GSP

Il s'agit de la seule anomalie qui soit bien prévisible d'après le schéma sur la figure 2. Le gène GSP code pour la sous-unité $\mathrm{G} \alpha$ s qui transmet le signal du récepteur de la TSH vers l'adénylate cyclase. Comme les autres protéines $\mathrm{G}$, celle-ci oscille entre deux états, en fonction du nucléotide fixé [26]. En l'absence de la TSH, G $\alpha_{\text {s }}$ fixe le GDP et adopte une conformation inactive. Le récepteur stimulé interagit avec le $\mathrm{G} \alpha_{\mathrm{s}}$, induit un échange du GDP par le GTP et par conséquent une transition vers l'état actif. Normalement, le signal se termine spontanément au moment de l'hydrolyse du GTP par la GTPase intrinsèque de $\mathrm{G} \alpha$. Cependant, des mutations ponctuelles spécifiques (au niveau des codons 201 ou 227) peuvent supprimer cette capacité GTPasique, déclenchant donc une activité constitutive de la voie AMP cyclique. Comme prévu, la plus grande incidence de ces mutations se trouve dans les " adénomes toxiques " [32], au niveau desquels existe une suractivation de cette voie, qui se traduit par une surexpression des fonctions différenciées (fixation de l'iode et sécrétion des hormones thyroïdiennes) surajoutée à l'augmentation de la croissance. Cependant, ces mutations ont été retrouvées aussi dans des tumeurs moins différenciées (bénignes ou malignes) dont les cellules ont plus ou moins perdu leur capacité de sécrétion d'hormones, mais ont évidemment conservé la réponse proliférative à l'AMPc [33]. (Dans ces cas, le phénotype prévisible - une augmentation du taux basal de l'activité adénylate cyclase - avait été déjà noté avant les analyses moléculaires [33].)

Le fait que les mutations GSP (au moins dans les codons 201 et 227) n'aient été retrouvées que dans la moitié des adénomes toxiques laisse supposer que le même phénotype pourrait être provoqué par l'activation de gènes codant pour d'autres éléments de la voie $\mathrm{AMPc}$, tels que le récepteur de la $\mathrm{TSH}$, où bien l'adénylate cyclase elle-même.

- Mutation des gènes RAS

Analogue à GSP, les trois petites protéines $G$ appartenant à la famille Ras représentent un switch moléculaire oscillant entre un état basal à GDP et un état actif à GTP (sans que l'on connaisse précisément les liens en amont et en aval) $\left(\mathrm{m} / \mathrm{s} n^{\circ} 5\right.$, vol. 8 , p. 471) [16]. Une mutation affectant les codons $12 / 13$ ou 61 active la protéine en supprimant sa capacité GTPasique. Il est probable que ces protéines $\mathrm{H}$-, $\mathrm{K}$ - et $\mathrm{N}$-Ras assurent la transmission des signaux de divers récepteurs à activité TK vers des kinases à sérine/thréonine (voir figure 2 et $\mathrm{m} / \mathrm{s} n^{\circ} 5$, vol. 8, p. 471). Les voies empruntées par Ras doivent être d'une importance capitale pour la cellule vésiculaire, car une mutation activatrice de l'un de ces gènes a été retrouvée dans près de $50 \%$ des tumeurs vésiculaires bénignes aussi bien que malignes [34, 35].

Par ailleurs, le rôle clé de Ras dans ces tumeurs a été démontré d'une façon frappante par des expériences de transfert de gène in vitro. L'introduction d'un gène Ras muté dans des cellules épithéliales en culture primaire, prélevées sur des thyroïdes normales humaines, provoque une réponse proliférative frappante avec une augmentation de la capacité reproductive de ces cellules de moins de trois divisions jusqu'à plus de 20 [36] (figure 3). Une telle stimulation, avec conservation de fonctions différenciées, mime le phénotype d'un adénome in vivo. Ces données, ainsi que l'observation de mutations RAS dans les tumeurs thyroïdiennes les plus précoces, suggèrent fortement qu'une telle mutation représente un événement initiateur de la tumorigenèse au niveau de la cellule vésiculaire. (Il faut attendre des expériences similaires avec GSP pour voir si ce dernier possède le même potentiel).

Activation de récepteurs - réarrangement des gènes RET et TRK

Ce sont des récepteurs à TK qui sont activés, comme beaucoup d'autres, 

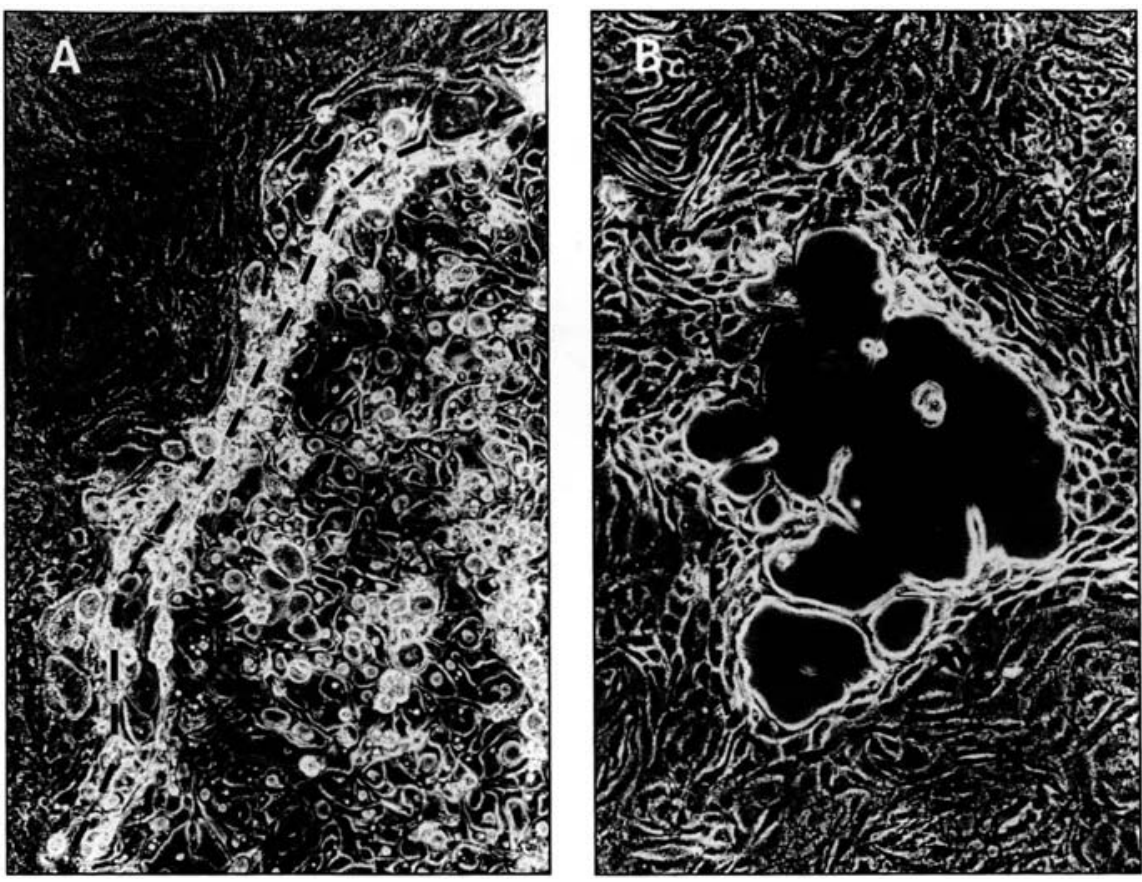

Figure 3. Preuve expérimentale de l'aptitude des oncogènes RAS et RET à déclencher une prolifération néoplasique thyrö̈dienne. Les oncogènes activés sont introduits dans des cellules épithéliales thyroïdiennes en culture primaire au moyen de vecteurs rétroviraux amphotropiques ([36] et en préparation). (A) Le gène RAS muté induit une forte prolifération, les colonies qui en résultent refoulant les cellules normales avoisinantes [N]. (B) RET activé provoque également une prolifération excessive, mais moins marquée et plus inhibée par les cellules adjacentes. De plus, les colonies RET développent des " trous " caractéristiques [T] entourés de cellules entassées, avec quelquefois des projections qui rappellent l'aspect d'un micro-cancer papillaire. (Pointillés : bordure de colonie ; contraste de phase $\times 125.1$

non par mutation ponctuelle, mais par un remaniement du gène. (TRK est un réccpteur du NGF (nerve growth factor) ; le ligand de RET reste incertain.) La protéine normalc comporte une partic $\mathrm{N}$-terminale extracellulaire qui (en l'absence du ligand) semblc inhiber l'activité du domaine TK intracellulaire. A la suite d'une cassure du gène, suivie d'une translocation ou d'une inversion chromosomique, la partie TK est séparée de la région inhibitrice en amont, qui est remplacée par la partie $\mathrm{N}$-tcrminale d'unc protéinc "neutre", et se trouve donc libérée du contrôle inhibiteur normal. Dans la thyroïde, on a trouvé des réarrangements de RET avec un gène de fonction inconnuc, nommé H4 [27], ct, plus réccmment, avec le gène d'une protéinc kinase A [37].

TRK est associé d'une manière similaire à deux gènes codant pour des protéines structurales, la tropomyo-

$\mathrm{m} / \mathrm{s} n^{\circ} 1$ vol. 9, janvier 93 réccpteurs particuliers, et n'impliquent pas d'autres réccpteurs à TK comme cclui de l'EGF. Par ailleurs, pourquoi sont-ils si spécifiques des tumeurs à ccllules vésiculaires et non pas dc celles développées à partir des ccllules $\mathrm{C}$ ? Est-il possible qu'il existe un système de "rétro-contrôle " qui limite le potentiel nocif d'un réceptcur constitutivement activé, mais qui n'opère que sur les récepteurs propres à la ccllule en cause ?

Des expéricnces de transfert de gène analogucs à ccllcs de RAS ont récemment démontré que RET activé (réarrangé avec $\mathrm{H} 4$ ) est aussi capable de provoquer directement unc hypcr-prolifération des cellules vésiculaires thyroïdiennes en culture primairc. Cependant, la morphologie des colonies qui en résultc diffère d'unc façon scnsible de cclle produite par un gène RAS muté, et la durée de la prolifération cst plus courte (J. A. Bond et D. Wynford-Thomas, cn préparation) (figure 3 ).

Inactivation des voies inhibitrices : anti-oncogènes inactivés

A la différence de nos connaissances asscz avancées concernant l'initiation de la néoplasie dans la thyroïde, les gènes impliqués dans la progression tumorale (adénome vésiculairc à cancer vésiculaire ; carcinome papillairc occulte à carcinome papillaire progressif) restent inconnus (figure 4). Un grand nombre d'oncogènes a déjà été étudié sans succès. D'après lcs analyscs d'autres tumcurs, les anomalies cn cause seraient l'inactivation d'un ou plusicurs gènes codant pour des éléments clés des voics inhibitrices de la prolifération, c'cst-à-dire des " antioncogènes ", dont quelques candidats peuvent être déjà postulés. Ainsi, la fréquencc élevée du canccr thyroïdien dans le syndrome de Gardner suggère un rôle éventuel d'unc mutation du gène $A P C$ (gène de susceptibilité à la poly-adénomatose colique familiale) [40]. Des travaux cytogénétiqucs et des analyses de l'hétérozygotie des tumcurs thyroïdiennes suggèrent l'existence d'un gène suppresseur putatif, localisé sur le bras court du chromosome 3 [41]. Rcste à noter qu'un des gènes candidats - p53 qui cst, globalement, la lésion moléculaire la plus fréquente dans les cancers humains [42], a été exclu de ce 


\section{RÉFÉRENCES}

28. Bongarzonc I, Pierotti MA, Monzini N, et al. High frequency of activation of tyrosinc kinasc oncogenes in human papillary thyroid carcinoma. Oncogene 1989; 4: 1457-62

29. Lemoine NR, Hughes CM, Brown CL, Wynford-Thomas D. Abnormalities of the EGF receptor system in human thyroid ncoplasia. Int J Cancer 1991; 49 : 558-61.

30. Lemoinc NR, Hughes CM, Brown CL, et al. Absence of abnormalitics of the ccrbB-1 and c-crbB2 proto-oncogenes in human thyroid neoplasia. Eur $J$ Cancer $1990 ; 26: 777-9$

31. Wyllic FS, Lemoinc NR, Williams ED, Wynford-Thomas D. Structure and expression of nuclear oncogenes in multistage thyroid tumorigenesis. $\mathrm{Br} J$ Cancer 1989 ; 60 : 561-5.

32. O'Sullivan C, Barton CM, Staddon SL, Brown CL, Lemoinc NR. Activating point mutations of the gsp oncogenc in human thyroid adenomas. Mol Carcinogen 1991; 4 : 345-9.

33. Suarcz HG, du Villard JA, Caillou B, Schlumberger M, Parmentier C, Monier R. gsp mutations in human thyroid tumours. Oncogene 1991; 6 : 677-9.

34. Suarez HG, du Villard JA, Scverino $\mathrm{M}$, et al. Presence of mutations of all threc ras genes in human thyroid tumours. Oncogene 1990; 5 : 565-70.

35. Lemoinc NR, Mayall ES, Wyllic FS, et al. High frequency of ras oncogenc activation in all stages of human thyroid tumorigencsis. Oncogene 1989; 4 : 159-164.

36. Lemoinc NR, Staddon S, Bond J, Wyllic FS, Shaw JJ, Wynford-Thomas D. Partial transformation of human thyroid cpithelial cells by mutant Ha-ras oncogene. Oncogene $1990 ; 5: 1833-7$.

37. Bongarzone I, Carcano C, Monzini N, et al. Ret activation in papillary thyroid carcinomas. Oncogenes and Growth Control. Heidelberg : EMBL, 1992 : 35.

38. Greco A, Picrotti MA, Bongarzone I, Pagliardini S, Lanzi C, Della Porta G. Trk$\mathrm{T} 1$ is a novel oncogenc formed by the fusion of tpr and trk genes in human papillary thyroid carcinomas. Oncogene 1992; 7 :

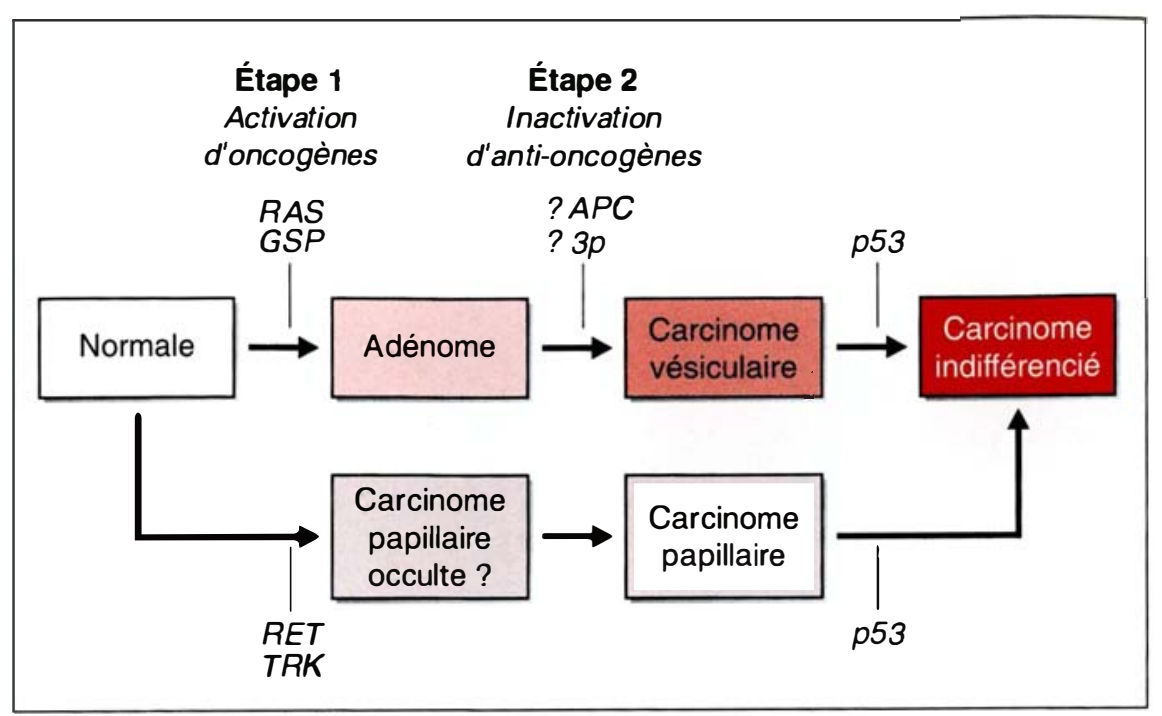

Figure 4. Genèse de la néoplasie dans la cellule vésiculaire : deux voies et de multiples étapes. Les observations anatomo-pathologiques ont individualisé deux voies divergentes de la tumorigenèse à partir de la cellule vésiculaire : l'une menant, semble-t-il directement, à des tumeurs malignes de type " papillaire" ; I'autre à des tumeurs bénignes ou malignes de type "vésiculaires". (Les arguments en faveur de la progression adénome-carcinome sont proposés ailleurs [50].) L'analyse de pièces opératoires ainsi que les expériences in vitro suggèrent que la "direction " de la tumorigenèse est déterminée par la nature de la lésion moléculaire initiatrice. II est intéressant que, dans les deux cas, il s'agit d'un oncogène activé, à la différence de la tumorigenèse colique, qui semble être déclenchée par la mutation d'un anti-oncogène (voir [48] et figure 7). Ce contraste dans l'ordre des événements pourrait refléter les cinétiques distinctes des deux tissus (figure 5). A noter : (1) La spécificité oncogène-tumeur dans ce schéma ne regarde que l'étape initiatrice. L'observation de mutations GSP et RAS survenant dans des cancers papillaires assez avancés [33] ne va donc pas à l'encontre de notre hypothèse ; (2) Ce schéma ne prétend pas être exhaustif, RAS, GSP, RET et TRK n'étant sûrement pas les seuls initiateurs possibles.

rôle dans la thyroïde, sauf dans l'étape la plus tardive (la transition d'un cancer différencié en un cancer anaplasique) [43, 44].

Schéma du déclenchement de la tumorigenèse dans un tissu à renouvellement conditionnel : la thyroïde Le schéma le plus simple pouvant intégrer toutes ces données est montré sur la figure 4. Les résultats des transferts géniques in vitro, en association avec les observations faites sur des échantillons de tumeurs, conduit à l'hypothèse que Ret/Trk et Ras/Gsp représentent deux classes d'initiateurs qui déclenchent la tumorigenèse thyroïdienne à travers des voies différentes : Ras/Gsp via un adénome vésiculaire progressant éventuellement vers un cancer de la même morphologie ; Ret/Trk, via de petites lésions papillaires ("carcinomes papillaires occultes"), vers un cancer papillaire cliniquement évident. On a donc, pour ce qui est de la thyroïde, une explication assez satisfaisante de l'initiation du processus néoplasique, qui, de plus, fournit un exemple frappant de la manière dont la nature de la lésion génique détermine l'aspect pathologique et le comportement clinique de différentes sortes de tumeurs provenant de la même cellule.

\section{Limites \\ du modèle thyroïdien pour les tumeurs d'un épithélium à renouvellement rapide : le modèle côlon}

Comme nous l'avons discuté plus haut, dans un tissu "stable " telle la thyroïde, où il n'y a pratiquement aucune mort cellulaire, une augmentation de la prolifération est suffisante pour assurer une croissance illimitée 
Figure 5. Influence de la cinétique du tissu normal sur la conséquence d'une altération du cycle cellulaire. Le schéma illustre le résultat d'une mutation survenant dans une seule cellule (noyau noir) et qui n'aurait pour effet que la stimulation de la prolifération cellulaire. Dans le côlon (B), cela serait la conséquence d'un raccourcissement de la durée du cycle cellulaire; dans la thyroide (A), cela pourrait aussi être dû à une augmentation de la proportion de la population qui est dans le cycle (growth fraction). Dans le cas (A), où il n'y a effectivement aucune mort cellulaire, une augmentation du taux de naissance d'un niveau près de zéro à un niveau significatif (" $X$ ») aboutira inévitablement à une croissance progressive du clone muté, c'est-à-dire à un néoplasme. En revanche, dans (B), l'excès de naissances par rapport aux morts sera automatiquement limité, tant que $50 \%$ des cellules filles se différencieront. Un nouvel équilibre sera établi, l'effet se bornant donc à une augmentation du nombre de cellules dans le compartiment différencié (c'est-à-dire, une hyperplasie).
Figure 6. Importance clé de la différenciation pour la stabilité d'un tissu à renouvellement rapide. Tandis qu'une simple accélération de la division des cellules souches ne provoque qu'une hyperplasie (figure 5), une perturbation de la différenciation a des conséquences beaucoup plus graves. Le schéma illustre une mutation (noyau noir) d'une cellule souche (hachurée) qui réduit la probabilité de différenciation de ses cellules filles à moins de 0,5. Par conséquent, après certaines mitoses, les cellules filles seront toutes les deux retenues dans le compartiment prolifératif, ce qui entrainera une croissance progressive du nombre de ces cellules et ainsi de la population totale - autrement dit, un néoplasme.

$\mathrm{m} / \mathrm{s} n^{\circ} 1 \mathrm{vol} .9$, janvier 93
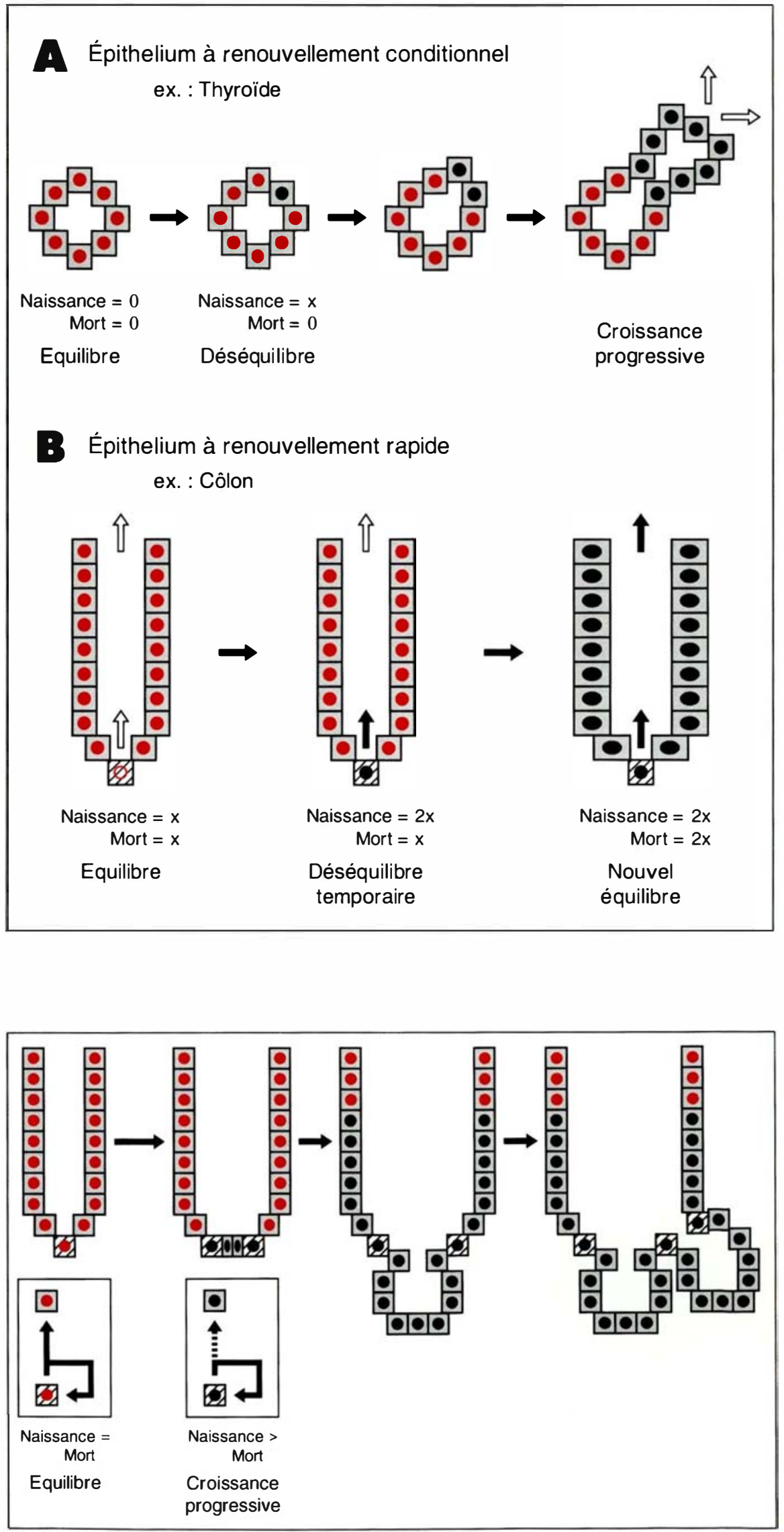


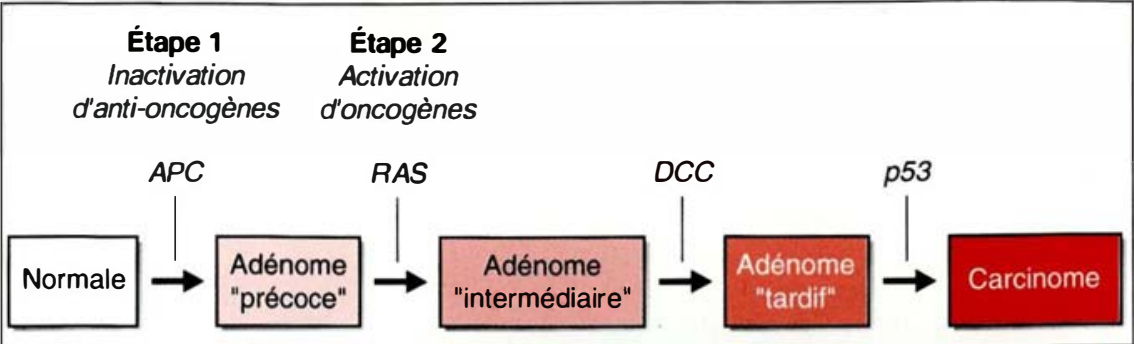

\section{RÉFÉRENCES}

39. Santoro M, Rosati R, Grieco M, et al. The ret proto-oncogene is consistently expressed in human pheochromocytomas and thyroid medullary carcinomas. Oncogene 1990 ; 5 : 1595-8.

40. Kinzler KW, Nilbert MC, Su I,K, et al. Identification of FAP locus genes from chromosome 5q21. Science 1991; 253 : 661-5.

41. Herrman MA, Hay ID, Bartelt DH, et al. Cytogenetic and molecular genetic studies of follicular and papillary thyroid cancers. J Clin Invest 1991 ; 88 : 1596-604.

42. Iscvine AJ, Momand J, Finlay CA. The p53 tumour suppressor gene. Nature 1991 . 351 : 453-6.

43. Wright PA, Lemoine NR, Goretzki P et al. Mutation of the p53 gene in a differentiated human thyroid carcinoma cell line, but not in primary thyroid tumours. Onco gene 1991 ; 6 : 1693-7.

44. Ito $T$, Seyama $T$, Mizuno $T$, et al. Unique association of $\mathrm{p} 53$ mutations with undifferentiated but not with differentiated carcinomas of the thyroid gland. Cancer Res 1992 ; 52 : 1369-71.

45. Fearon ER, Vogelstein B. A genetic model for colorectal tumorigenesis. Cell $1990 ; 61: 759-67$

46. Powell SM, Zilz N, Beazer-Barclay Y, et al. APC mutations occur carly during colorectal tumorigenesis. Nature 1992 ; 359 : 235-7.

47. Fearon ER, Cho KR, Nigro JM, et al. Identification of a chromosome $18 \mathrm{q}$ gene that is altered in colorectal cancers. Science $1990 ; 247: 49-56$

48. Kinzler KW, Nilbert MC, Vogelstein $\mathrm{B}$, et al. Identification of a gene located at chromosome $5 \mathrm{q} 21$ that is mutated in colorectal carcinomas. Science 1991; 251 : 1366-70.

49. Bos JL, Fearon ER, Hamilton SR, et al. Prevalence of ras gene mutations in human colorectal cancers. Nature 1987 $327: 293-7$

50. Wynford-Thomas D. Thyroid cancer In : I,emoine NR, Cooke T, Neoptolemos J, cds. Genes, Cancer and the Surgeon. Oxford :

Figure 7. Tumorigenèse dans I'épithélium du côlon. Comme dans la thyroïde (figure 4), il y a plusieurs étapes histologiquement individualisées, et associées plus ou moins étroitement à des anomalies géniques. Bien que l'ordre puisse varier dans les stades avancés, on peut déceler un contraste potentiellement instructif avec la thyroìde en ce qui concerne l'étape d'initiation. Dans le côlon, le premier événement semble impliquer un anti-oncogène, suivi souvent par l'activation d'un oncogène, alors que dans la thyroïde, le processus est inversé, commençant obligatoirement avec l'activation $d^{\prime} u n$ oncogène. (D'après Vogelstein [45-48].)

- autrement dit, une tumeur (figure 5). En revanche, dans un tissu à renouvellement rapide comme le côlon, la figure 5 montre qu'un simple accroissement du taux de naissance des cellules n'entraînera qu'une augmentation restreinte de la population cellulaire. Certes, le taux de production de cellules restcra élevé cn permanence, mais cela sera compensé par une élévation équivalente du taux de mort ccllulairc, lc résultat final étant seulement un épithélium hyperplasique. Pour qu'une véritable tumeur se développe, l'essentiel est non pas un changcment du temps dc doublement de la cellule souche, mais une altération de la proportion de ses cellules filles qui sont commises à la différenciation "terminale " (figure 6). C'est ce rapport (normalement fixé à égalité) entre la prolifération et la mort qui assure la constance de la population souche et donc empêche une croissance exponentielle.

Le problème actuel est l'état limité de nos connaissances des mécanismes qui sous-tendent lc processus de différenciation. Il est possible que le comportement normalcment différent des deux cellules filles soit dû à une asymétrie intrinsèque survenant lors de la division de la cellulc souche. Il pourrait être le résultat automatique d'un simple effet topologique, la cellule fille qui se trouve expulsée de la " niche" privilégiée de la zone souche recevant des signaux de différenciation du stroma voisin. Quoi qu'il en soit, on peut donc s'attendre à ce que l'anomalie crucialc pouvant déclencher une tumeur dans un tel tissu soit non pas une hyperstimulation des voies mitogènes, mais une inhibition des signaux qui assurent habituellement la différenciation (et donc finalement la mort) de $50 \%$ des cellules filles.

Anomalies génétiques dans la tumorigenèse du côlon : comparaison avec la thyroïde

Les tumeurs du côlon étant beaucoup plus fréquentes que celles dc la thyroïde, elles ont été plus intensément étudiées, et les travaux de B. Vogelstein ont permis de proposer un modèle détaillé concernant la progression tumorale dans cc tissu [45, 46] (figure 7). En particulier, la relative abondance des échantillons coliques par rapport aux tumeurs thyroïdiennes a facilité les analyses chromosomiques (cytogénétiques et moléculaircs) qui ont apporté une connaissance beaucoup plus avancée des anti-oncogènes impliqués dans la progression de la tumorigenèsc colique [40, 45-48].

Néanmoins, en ce qui concerne les étapes précoces, une comparaison cntrc les tumeurs thyroïdiennes ct coliques s'avère fort intéressante. Lc côlon révèle une séquence adénomecarcinome analogue à la néoplasie vésiculaire (mais non papillaire) de la thyroïde. Des oncogènes ras mutés sont impliqués avec une grande fréquence dans les adénomes coliques [49] comme dans ccux de la thyroïdc $[34,35]$, et ces dcux exemples constituent les premières mises en évidence de ces anomalies dans l'étape bénigne d'une tumcur multi-ćtapc 
chez l'homme. Il existe cependant unc différence sensible, mais potentiellement très significative : dans la thyroïde, toutes les donncees impliquent une mutation RAS dans les toutes premières ćtapes (mutations retrouvées dans les adénomes et nodules censés être les lésions les plus précoces, cela est confirmé par les expériences de transfert du gène dans les cellules en culture). En revanche, dans le côlon, cet événement survient à une étape nettement plus tardive. D'après les analyses de Vogelstein $[45,46]$, la mutation du gène RAS serait rare dans les adénomes les plus précoces. Si ce résultat est confirmé (cette conclusion suppose un rapport taille de la tumeur/étape de la transformation qui n'a pas été strictement prouvé), il indique que la mutation de RAS doit être précédée par d'autres événements et que celle-ci ne serait donc pas capable, à elle seule, de déclencher la tumorigenèse dans ce tissu. Cette conclusion est tout à fait compatible avec les prévisions fondées sur les arguments cytocinétiques évoqués ci-dessus. Malheureusement, un test direct de cette hypothèse par transfert du gène RAS muté dans des cellules normales du côlon n'a pas été encore rapporté.

Le véritable initiateur pour le côlon reste incertain, mais les travaux de Vogelstein suggèrent fortement le rôle des gènes d'une nouvelle famille $\mathrm{APC} / \mathrm{MCC}[40,48]\left(\mathrm{m} / \mathrm{s} n^{\circ} 7\right.$, p. $718 ; n^{\circ} 6$, vol. 8, p. $607 ; n^{\circ} 9$, vol. 8, p. 1000). Encore en accord avec nos arguments, ce sont des antioncogènes, dont les protéines ont une homologie partielle avec les domaines cytoplasmiques de certaines classes de récepteurs. On peut supposer que si ces protéines transmettent les signaux responsables de la différenciation des cellules filles coliques, leur perte pourrait conférer à une telle cellule et à sa descendance les propriétés d'une cellule souche, c'est-à-dire la possibilité de continuer de proliférer et de s'accrocher à la membranc basale des cryptes coliques, ce qui lui évite le transport vers la surface luminale.

Après avoir contourné la limitation imposée par le mécanisme "autorégulateur " de l'asymétrie des cellules filles, la tumeur précoce serait effectivement arrivée à un état cinétique quasi équivalent à cclui où se trouve la cellule thyroïdienne en état normal. Cela expliquerait donc facilement que la prochaine étape soit une mutation de RAS.

\section{Conclusion}

Même s'il manque toujours les preuves définitives pour certaines de ces idées, celles-ci sont des exemples d'un mode de pensée qui deviendra de plus en plus important si on veut profiter au mieux des avancées spectaculaires de l'oncologie moléculaire fondamentale. Les bénéfices d'une telle approche intégrative sont en effet réciproques - à la fois pour le fondamentaliste mais aussi pour le clinicien/pathologiste. D'un côté, la connaissance des modes d'action d'un oncogène peut expliquer au clinicien le comportement ou la morphologic d'une tumeur, mais, d'autre part, une bonne connaissance du tissu, de l'organe peut orienter les travaux du fondamentaliste et même lui suggérer des expériences originales!

\section{Summary}

Origin and progression of epithelial tumours : cellular and molecular mechanisms

More than $90 \%$ of cancers in man are of epithelial origin. Recent technological advances, notably the advent of PCR, have led to a rapid expansion in our knowledge of the somatic genetic abnormalities in such tumours, including some of the most clinically important examples (breast, lung and colon). However, a list of genetic lesions does not by itself provide an explanation of tumour behaviour at the biochemical and cellular levels. This review illustrates the need to address the interface between molecular genetics and classical tumour pathology, by comparing and contrasting the molecular and cellular biology of tumorigenesis in two model epithelia - thyroid and colon. The influence of normal cell kinetics on the predicted sequence of molecular events provides a good example of the importance of tissue context in molecular oncology.

\section{Remerciements}

Je remercie le professeur Thierry Soussi et le docteur Corinne Gérard pour leurs commentaires et leur lecture critique du manuscrit. 